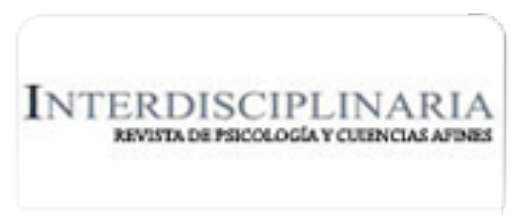

Interdisciplinaria

ISSN: 0325-8203

ISSN: 1668-7027

interdisciplinaria@fibercorp.com.ar

Centro Interamericano de Investigaciones Psicológicas y

Ciencias Afines

Argentina

Cataldo, Rocio; Liberatore, Gustavo; Hermosilla, Ana María

Aplicación de la Ley 26.657 de Salud Mental: una mirada desde la formación de psicólo $g o s$ en la Universidad Nacional de Mar del Plata

Interdisciplinaria, vol. 35, núm. 2, 2018, Julio-, pp. 277-289

Centro Interamericano de Investigaciones Psicológicas y Ciencias Afines

Argentina

Disponible en: https://www.redalyc.org/articulo.oa?id=18058785003

Cómo citar el artículo

Número completo

Más información del artículo

Página de la revista en redalyc.org

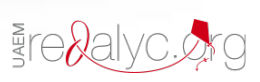

Sistema de Información Científica Redalyc Red de Revistas Científicas de América Latina y el Caribe, España y Portugal Proyecto académico sin fines de lucro, desarrollado bajo la iniciativa de acceso abierto 


\title{
Aplicación de la Ley 26.657 de Salud Mental: una mirada desde la formación de psicólogos en la Universidad Nacional de Mar del Plata
}

\section{Application of Mental Health Law 26,657: a view from the training of psychologists at the National University of Mar del Plata}

\author{
Rocío Cataldo*, Gustavo Liberatore**, Ana María Hermosilla***
}

\begin{abstract}
*Licenciada en Psicología. Diplomada en Políticas en Salud Mental y Derechos Humanos. Becaria de Investigación, Categoría B, Universidad Nacional de Mar del Plata (UNMdP). Docente de la Cátedra de Deontología Psicológica de la Facultad de Psicología (UNMdP). E-mail: rociocataldo@hotmail.com

**Licenciado en Bibliotecología y Documentación. Doctor en Documentación. Profesor Titular en el Departamento de Documentación (UNMdP). Docente e Investigador (UNMdP). Codirector del grupo de investigación Psicología y Ética: Ciencia y Profesión. Facultad de Humanidades (UNMdP). E-mail: gliberat@mdp.edu.ar

***Licenciada en Psicología. Docente e Investigadora de la Universidad Nacional de Mar del Plata (UNMdP). Docente adjunta de la cátedra de Deontología Psicológica de la Facultad de Psicología (UNMdP). Directora del grupo de investigación Psicología y Ética: Ciencia y Profesión. E-mail: ahermo@mdp.edu.ar
\end{abstract}

Universidad Nacional de Mar del Plata. Buenos Aires, Argentina.

\section{Resumen}

En función de las nuevas formas de concebir las prácticas profesionales en el área de la salud mental, tendientes al reconocimiento de derechos de los pacientes, la formación de recursos humanos, adquiere un papel fundamental. En Argentina, a partir de la sanción de la Ley Nacional de Salud Mental 26657, la formación de profesionales, incluyendo los psicólogos, aparece como un requisito central para su aplicación efectiva. La temática tiene relación con tres niveles fundamentales: el legal, él ético y el deontológico. En este marco, se propone un estudio de tipo exploratorio-descriptivo sobre la formación de psicólogos en la Universidad Nacional de Mar del Plata, en relación a los principios, políticas y dispositivos establecidos en cumplimiento de la Ley de Salud Mental 26.657.

Palabras clave: Salud mental; Ley 26.657; Formación; Psicología; Psicólogos.

\begin{abstract}
New ways of conceiving practices in the area of health have been manifested in recent years with the enactment of a series of laws that modify professional practices. The National Law 26.657 (LSM) represented this change specifically in the area of Mental Health. Both the conceptualization of notions such as mental health, diagnosis, true and imminent risk, interdis- cipline, as well as the proposed approach, tend towards the recognition and respect of the rights of patients. That is, a central place in the treatment of physical illnesses and mental disorders is given back to subjectivities. In this context of change, the formation of human resources, including psychologists, appears as a central requirement for its effective application. Formation, in a broad sense, includes not only the specific knowledge that psychologists must possess, but also values and a vision of man as a subject of law. The theme is related to three fundamental levels: legal, ethical and deontologi-
\end{abstract}


cal. In this case, a study on the training of psychologists at the National University of Mar del Plata (UNMdP) is proposed, in relation to the principles, policies and devices established in compliance with the Mental Health Law 26.657. The investigation was of a qualitative-quantitative type with a transversal non-experimental design of exploratory-descriptive type. The methodology used included, in the first place, the documentary analysis of the teaching programmes of the subjects that make up the course curriculum. Thirty-one subjects in the curriculum of the Psychology Department of the UNMdP were considered for the analysis and a data structure was designed that contemplated the different aspects from which a bibliographic corpus can be analyzed taking into account the set of variables necessary for a coherent representation of the field. Finally, ten categories were created for the analysis of the programs. They arise from the nodal chapters and themes in the Law: (1) Interdisciplinarity, (2) Human Rights (3) Law of Mental Health / Mental Health (4) Community Psychology (5) Institutional Psychology (6) Patients (7) Disability (8) Drug (9) Devices (10) Standards and Treaties: in human rights and mental health. The second phase consisted in the application of a quantitative instrument, with the purpose of collecting information as regards the opinion of the students in the last year of the course of study with respect to their training. The sample was non-probabilistic of incidental type of anonymous and voluntary participation. The questionnaire was administered to 48 students who were enrolled in the last year of the Psychology Degree at the National University of Mar del Plata. An instrument consisting of a questionnaire combined with a Semantic Differential Scale was constructed, and the questionnaire was administered to students who were specifically studying one of the last 4 subjects: Clinical Psychology, Educational Psychology, Labor Psychology or Legal Psychology. Informed consent was obtained from the explanation of the objectives of the research, and the voluntariness of the participation and the disposition of the results for those who want to consult them was clarified. It is expected that the contributions of the study carried out are significant in order to analyze the situation of our university course in relation to the subject. The research seeks to represent a first diagnostic approach to what the situation in the course of Psychology in the UNMDP is, to know the reality from which it starts, and on which the new contributions and challenges will be built.

Keywords: Health Mental; Law 26657; Training; Psychology; Psychologists.

\section{Introducción}

Las nuevas formas de concebir las prácticas en el área de la salud, se han manifestado en los últimos años, en la sanción de una serie de leyes que modifican las prácticas profesionales. La Ley Nacional 26.657 (LSM) representó este cambio específicamente en el área de la Salud Mental.

Restrepo Espinosa (2006) refiere a este nuevo paradigma a través del cual deben orientar su quehacer los profesionales de la salud, y en especial los de la salud mental. Destaca como uno de los principales cambios, el reconocimiento del impacto de lo social y lo institucional en la salud individual, y el planteo de que esta última debe ser pensada a través de conceptos como bienestar, desarrollo humano y calidad de vida.

El eje conductor de los cambios mencionados es la centralidad otorgada a los derechos de las personas. Es decir, se le devuelve a las subjetividades, un lugar central en el tratamiento de las enfermedades físicas y los padecimientos mentales.

En un estudio realizado con anterioridad en la ciudad de Mar del Plata (Hermosilla \& Cataldo 2014), se ha observado que una de las principales dificultades para la implementación de la LSM, refiere a la necesidad de que la formación de los profesionales de las distintas disciplinas sea acorde con los desafíos planteados. Formación en un sentido amplio, incluyendo no solo los conocimientos específicos que los psicólogos deben poseer, sino también valores y una visión del hombre como sujeto de derecho.

El artículo 33 de la LSM (2010) aborda la temática de la siguiente manera: "La Au- 
toridad de Aplicación debe desarrollar recomendaciones dirigidas a las universidades públicas y privadas, para que la formación de los profesionales en las disciplinas involucradas sea acorde con los principios, políticas y dispositivos que se establezcan en cumplimiento de la presente ley, haciendo especial hincapié en el conocimiento de las normas y tratados internacionales en derechos humanos y salud mental. Asimismo, debe promover espacios de capacitación y actualización para profesionales (...)".

En el año 2014, la Comisión Nacional Interministerial en Políticas de Salud Mental y Adicciones elaboró, a través de un proceso de consulta y discusión con diversos actores, las Recomendaciones a las universidades públicas y privadas para la formación. Este documento cuenta con tres apartados fundamentales: Enfoque de derechos, Inclusión social, e Interdisciplina, como ejes transversales a ser considerados en la formación de los profesionales.

Existen otros instrumentos, de carácter internacional, en los que se encuentra plasmada la mencionada necesidad de reconvertir el perfil profesional; por un lado la Declaración de Caracas (1990), que estipula que la capacitación del recurso humano en Salud Mental y Psiquiatría debe hacerse apuntando a un modelo cuyo eje pase por el servicio de salud comunitaria y propicie la internación psiquiátrica en los hospitales generales. Por otro lado el Consenso de Panamá (2010), insta a los Estados miembros de la Organización Panamericana de la Salud, a que consideren el fortalecimiento de los recursos humanos en materia de Salud Mental como un componente clave del mejoramiento de los planes y servicios.

Por último, la Convención de las Personas con Discapacidad establece la responsabilidad de: "Promover la formación de los profesionales y el personal que trabajan con personas con discapacidad respecto de los derechos reconocidos en la presente Convención, a fin de prestar mejor la asistencia y los servicios garantizados por esos derechos" (art. 4.1 inciso i, 2006).
Entre los autores que han trabajado la temática, Rosendo (2011), considera la formación de recursos humanos como una variable determinante, siendo necesario un perfil y un desempeño profesional acorde a las necesidades de la transformación que dictamina la Ley. La autora sostiene que esta línea podría ser un serio obstáculo para su viabilidad.

Rosendo ya había planteado la necesidad de reconversión profesional, en relación a la Ley de Salud Mental de la ciudad de Buenos Aires ( $\left.\mathrm{n}^{\circ} 448\right)$, lo cual podría extenderse a la reciente ley nacional. La autora establece en su trabajo una serie de particularidades del perfil de los profesionales de la salud mental, a saber: orientación hacia tratamientos individuales en detrimento de los grupales y familiares; escasa experiencia en intervención en situaciones de crisis; insuficientes actividades en promoción de la salud, prevención de la enfermedad y en rehabilitación; escasa formación en abordajes comunitarios; dificultades para planificar tratamiento y delimitar objetivos en tiempos definidos; desconocimiento de la epidemiología actual de salud mental en la ciudad; actividades docentes orientadas predominantemente a la clínica psicoanalítica $\mathrm{y}$ escasa actividad investigativa.

Según Borgioli y otros (2011), el desafío en relación a la LSM involucra a la formación de grado ya que plantea modificaciones que abarcan a las incumbencias profesionales a la vez que prescribe acciones que requieren replantear las prácticas.

De Lellis (2011) ha abordado la cuestión de la formación de profesionales a partir de la Ley. Entre sus aportes, se puede mencionar la exposición de algunas experiencias pedagógicas que ilustran la importancia de promover prácticas pre-profesionales que faciliten la formación de roles indispensables para el futuro quehacer profesional.

Aunque ha trabajado sobre la formación de profesionales en el ámbito de la Salud en general, Moran-Barrios (2013) destaca la necesidad de definir cuál es el perfil profesional al que se apunta, y que éste se en- 
cuentre adaptado a las necesidades sociales y del sistema sanitario. El autor cita dos informes referidos a la formación de médicos, que marcan un lineamiento general similar al planteado en este trabajo sobre el cambio de paradigma en Salud Mental. Tanto el informe del Hasting Center como el de la Comisión Education of Health Professionals for the 21 Century marcan que la formación de profesionales debe virar desde el enfoque en la enfermedad, hacia una visión más holística y sistémica. Se destaca la necesidad de currículas basadas en el mejoramiento del rendimiento de los sistemas de salud mediante la adaptación de las competencias profesionales a los contextos específicos.

En cuanto a investigaciones empíricas en la temática, existen las que se han centrado en la perspectiva de los profesionales respecto a la ley, aunque no es equivalente el desarrollo en la temática de la formación universitaria. Aunque no versa específicamente sobre la LSM, Más (2010), realizó un estudio descriptivo en la ciudad de Mar del Plata entre los años 2006 y 2009, sobre la formación de psicólogos para el ejercicio profesional en salud pública, lo cual se encuentra vinculado con la temática a tratar. Se llevaron a cabo tres relevamientos principales en esta investigación. El primero de tipo documental respecto a los planes curriculares, programas sanitarios y/o resoluciones fundantes de los servicios de salud pública. El segundo, entrevistas a informantes calificados seleccionados por muestreo teórico y que fueran autoridades de las unidades académicas, responsables de prácticas curriculares relacionadas con la temática o autoridades sanitarias del sistema de salud local. Por último, una encuesta de relación educación-trabajo a estudiantes avanzados de cuatro carreras seleccionadas. El mencionado autor destaca que es necesaria una reorganización profunda de los servicios de salud pública y del perfil de competencias requeridas para ese trabajo.

Por su parte, Zaldúa et al. (2011), a partir de una investigación realizada, plantean la formación profesional como requisito para la aplicación de la Ley. La investigación tuvo por objetivo comprender las representaciones y significaciones de los trabajadores del sector acerca de la viabilidad de la Ley 26.657 y sus efectos. Con este propósito, se confeccionó un cuestionario con preguntas abiertas para desarrollar. La muestra fue intencional estratificada por profesiones y estuvo formada por trabajadores interesados en la temática de salud mental que tenían conocimiento de la Ley en cuestión.

D`Agostino (2015) realizó una investigación con el objetivo de analizar la fundamentación, contenidos y bibliografía de los programas correspondientes al ciclo de formación superior en la carrera Licenciatura en Psicología (4to, 5to, 6to año) de la Universidad Nacional de La Plata, en función de ciertas categorías relacionadas con la LSM. Se establecieron las siguientes categorías de análisis: a) Salud Mental b) Interdisciplina c) Comunidad d) Derechos Humanos e) Adicción f) Políticas Públicas/ Sociales g) Prevención y h) Inclusión social. Se procedió a identificar y registrar en los programas, la presencia/ausencia de cada una, como así también la frecuencia en la presencia de las categorías.

A partir de lo expuesto, se observa en la bibliografía existente el planteo de la necesidad de reconversión del perfil profesional. Existen escasas investigaciones referidas a la temática, considerándose necesario el desarrollo de aquellas que reflejen la realidad local.

\section{Material y método}

La investigación es de tipo cuali-cuantitativa con un diseño no experimental transversal de tipo exploratorio-descriptivo. La metodología empleada consistió, en un primer momento, en la aplicación del análisis documental (contenido) a los diferentes registros bibliográficos seleccionados y su correspondencia a las categorías temáticas elegidas. En el segundo caso, fue el análisis 
resultante en la aplicación de un modelo de encuesta, que se desarrolló en dos fases.

Fase 1. La primera de tipo documental, se basó en el análisis de los programas de las asignaturas del plan de estudio de la carrera de Psicología (UNMdP). Esta fase del diseño metodológico se orientó a la evaluación curricular desde una perspectiva empírica, a través de la utilización de un análisis descriptivo de vertiente cuantitativa, aplicado a las referencias bibliográficas correspondientes a cada una de las asignaturas. Como otro indicador relevante para el análisis se calculó la vida media (VM) de los registros.

Se tomaron para el análisis 31 materias de la currícula de la carrera de Psicología de la UNMdP. Ésta se divide en cinco áreas, entendidas como unidades educativas funcionales, constituidas sobre la base de campos afines del conocimiento: Área de investigación en Psicología; Área socioantropológica; Área de sistemas psicológicos; Área psicobiológica; Área de ámbitos de trabajo psicológico.

En el análisis se excluyeron los siguientes requisitos curriculares, por no considerarse asignaturas dentro del plan y tener un régimen de cursada y estructuración diferente: Núcleos Problemáticos, Idiomas, GRAP, Seminarios de Orientación, Residencia, Trabajo de investigación final y Prácticas Preparatorias para la Práctica Profesional.

Se diseñó una estructura de datos que contempló los diferentes aspectos desde los cuales puede analizarse un corpus bibliográfico teniendo en cuenta el conjunto de variables necesarias para una representación coherente del campo. En dicha estructura, y a los fines de este estudio, fueron consignados los siguientes campos: Autor/es, Título, Año, Palabras clave, Asignatura/s (a la/s que pertenece el registro) y Categoría/s de análisis (de pertenencia).

Como se mencionó, el artículo 33 plantea que la formación de profesionales debe ser acorde a los principios, políticas y dis- positivos planteados en la ley. A partir de su lectura (y por no disponer al momento de comenzar la investigación del documento de Recomendaciones), se construyeron $10 \mathrm{ca}-$ tegorías para el análisis de los programas que surgen de los capítulos y temáticas nodales en la Ley:

1. Interdisciplina; 2. Derechos Humanos: pacientes como sujetos de derechos; 3 . Ley de Salud Mental/Salud Mental: Salud Mental como área, atención primaria de la salud y conocimiento de la Ley de Salud Mental; 4. Psicología Comunitaria: Salud Mental Comunitaria; 5. Psicología Institucional: en relación a las condiciones para ocupar cargos de conducción y gestión de servicios e instituciones; 6. Pacientes: derechos de los pacientes; 7. Discapacidad: cuestión de la capacidad/incapacidad; 8 . Fármaco: psicofarmacología; 9. Dispositivos: dispositivos alternativos como consultas ambulatorias, servicios de inclusión social y laboral, apoyo a personas y a las comunidades, casas de convivencia, hospitales de día, cooperativas de trabajo, centros de capacitación socio laboral; 10 . Normas y Tratados: en derechos humanos y salud mental.

Aquellas materias cuyos contenidos refieren específicamente a alguna variable, no fueron consideradas. En el caso de las variables Psicología Institucional y Psicología Comunitaria se excluyó la asignatura Psicología Institucional y Comunitaria, y en el caso de Normas y Tratados, se excluyó la asignatura Deontología Psicológica. Esto se hizo debido a que considerarlas incrementaría el número de registros bibliográficos en las variables correspondientes, sin ser representativo de la formación general.

El total de registros bibliográficos relevados fue de 1159 correspondientes a la bibliografía obligatoria de las asignaturas objeto de análisis. De este universo fueron extraídos 111 registros (cerca del 10\%) en los que se observó algún tipo de relación con las categorías temáticas de análisis. Para relacionar cada uno de los registros con las categorías temáticas seleccionadas se tomaron en cuenta dos aspectos: su per- 
tinencia mediante un análisis de su contenido $\mathrm{y}$, en los programas que lo indicaban explícitamente, la relación con ejes de abordaje relativos a dichas categorías.

Fase 2. La segunda fase consistió en la aplicación de un instrumento de tipo cuantitativo, con el objeto de recolectar información referida a la opinión de los estudiantes del último año de la carrera respecto a su formación.

La muestra fue no probabilista de tipo incidental, de participación anónima y voluntaria. Se administró el cuestionario a 48 estudiantes que se encontraban cursando el último año de la carrera de Psicología de la Universidad Nacional de Mar del Plata.

Se construyó un instrumento de tipo cuantitativo, consistente en un cuestionario combinado con una Escala de Diferencial Semántico. Dicha escala fue adoptada con el objeto de brindar una mayor elección de alternativas (amplitud de la escala bipolar) que permita ponderar adecuadamente y con suficiente amplitud la respuesta dada a los puntos requeridos y captar con mayor precisión la percepción del encuestado. Se buscó indagar la opinión de los alumnos respecto a la inclusión o no de ciertas temáticas en la formación.

Una vez construido el cuestionario, se realizó consulta a expertos, para recibir sugerencias y correcciones.

Luego se administró el cuestionario a estudiantes que estuvieran cursando el último año de la carrera, específicamente alguna de las últimas 4 asignaturas: Psicología Clínica, Psicología Educacional, Psicología Laboral o Psicología Jurídica.

Se obtuvo el Consentimiento Informado a partir de la explicación de los objetivos de la investigación y se aclaró la voluntariedad de la participación y la disposición de los resultados para quienes quieran consultarlos.

\section{Resultados}

\section{Fase 1}

En primer lugar, puede tomarse el análisis del peso que posee cada dimensión analizada en la totalidad de la currícula. La temática que mayor peso tiene es la de Salud Mental. Se debe tener en cuenta que ésta es abarcativa, ya que incluye diversas cuestiones referidas a la Salud Mental como área, atención primaria de la salud, y conocimiento de la Ley de Salud Mental (Figura 1).

Interdisciplina, Derechos Humanos y Psicología Institucional son temáticas con un alto peso en comparación con el resto, siendo de gran importancia en el marco de la Ley de Salud Mental.

Pacientes y Dispositivos poseen menor peso siendo dos categorías muy importantes ya que por un lado, Pacientes se relaciona con sus derechos, y por otro lado, Dispositivos hace alusión a aquellos necesarios para la implementación efectiva de la Ley.

Normas/Tratados posee bajo peso, lo cual es consistente con el bajo peso de $\mathrm{Pa}-$ cientes. Esto se debe a que es en las normas $\mathrm{y}$ tratados internacionales en derechos humanos y salud mental donde se expresan los derechos de los pacientes y se establecen los instrumentos para protegerlos (Historia Clínica, Secreto Profesional, Consentimiento Informado).

Fármaco es otra de las temáticas con escaso peso. Debe recordarse que el artículo 12 de la LSM plantea que debe promoverse que los tratamientos psicofarmacológicos se realicen en el marco de un abordaje interdisciplinario.

Por último, Discapacidad es la categoría con menor peso.

Realizando el análisis en función de las áreas curriculares (Figura 2), el área socioantropológica, posee el mayor número de referencias vinculadas a las variables. Le sigue el área de Ámbitos psicológicos que, sin embargo, posee poco más de la mitad de referencias que el anterior. 
Más allá del número de referencias, se encontraron registros correspondientes a la variable Salud Mental en todas las áreas de formación. Fue la única variable que presentó esta característica.

El área Psicobiológica fue la que menos cantidad de registros bibliográficos relacionados presentó, y sólo de las variables Salud Mental y Fármaco.

El área de Ámbitos psicológicos, presentó registros correspondientes a todas las variables, a excepción de Psicología Comunitaria y Fármaco. Esto es llamativo, teniendo en cuenta que el objetivo del área: es proveer al alumno una capacitación que le permita desempeñarse en los distintos campos de la Psicología Profesional. El ámbito comunitario, es uno de aquellos campos donde el psicólogo puede desempeñarse y actualmente, de gran importancia.

Otro dato a considerar, es la vida media de las referencias en análisis (Figura 3). Cuatro de las variables más representativas del espíritu de la Ley, Salud Mental, Psicología Comunitaria, Derechos Humanos e Interdisciplina, evidencian registros bibliográficos cuya vida media es de 15 años o más. Considerando la importancia y el desarrollo de estas temáticas en los últimos años, la bibliografía podría no estar actualizada.

Por otro lado, aquellas referencias con una vida media inferior, corresponden a dimensiones que de por sí son novedosas. Por ejemplo la dimensión Pacientes, la cual ha adquirido un énfasis particular los últimos años en el marco del paradigma de la Autonomía, el cual considera a los pacientes esencialmente como sujetos de derecho.

\section{Fase 2}

En cuanto a la opinión de los estudiantes próximos a recibirse, puede observarse que en líneas generales, existe una tendencia a considerar que las temáticas analizadas se incluyen escasamente (Figura 4).

En relación a cada una, se observa que:

- La temática del Consentimiento Informado es incluida en la formación, aunque las respuestas indican que se considera que su inclusión es de escasa a media.

- En relación a la temática de Historia Clínica, el $40 \%$ de los participantes consideran que no se incluye nada en la formación y otro $40 \%$ consideró que se incluye poco o muy poco. Es decir que, en líneas generales, es una temática considerada como escasamente abordada.

- En cuanto a la Interdisciplina, alrededor del $80 \%$ de los participantes consideró que la inclusión de esta temática es escasa. En consonancia con esto, puede observarse que el $80 \%$ consideró que la temática Conformación de Equipos Profesionales, no se incluye, o que se hace escasamente.

- Tanto en relación a las Internaciones en Salud Mental como en relación a la Psicofarmacología, alrededor del 90\% consideró que las temáticas no se incluyen, o que se incluye poco en la formación.

- Diagnósticos diferenciales, como Psicopatología, fueron temáticas que, en líneas generales, se acuerda en que se incluyen en la formación.

En cuanto a la inclusión de la cuestión de los Derechos Humanos en la formación, hubo acuerdo en que la temática se incluye (el 90\% coincidió en esto) (Figura 5).

Sin embargo, existen dos vías principales de inclusión. Una se relaciona con contenidos y bibliografía específica (analizado en la primera fase de la investigación), a través de valores, y de lo que se coincide en llamar cultura institucional, propia de cada institución de formación superior.

Las diferencias se observaron en relación a la medida en que se incluye la temática. Es decir, que si bien hubo acuerdo en que la cuestión de los Derechos Humanos es parte de la formación, la inclusión sería escasa. Por otro lado, se transmitiría en mayor medida través de valores, desde lo discursivo, y no desde contenidos formales. Esto indica que la temática de Derechos Humanos pertenece principalmente a lo que se conoce como currícula oculta, aquello que es transmitido en la formación por fuera de los contenidos formales que se incluyen en la planificación. 
En la Figura 6, puede observarse lo expuesto, a través de la relación entre la opinión de los alumnos respecto a la forma de inclusión de la temática Derechos Humanos y respecto a la medida en la cual se incluye.

\section{Consideraciones finales}

A nivel general, se observa en el análisis de programas, que solo alrededor del $10 \%$ de los registros analizados presentó algún tipo de relación con las categorías temáticas de análisis. Este porcentaje es bajo teniendo en consideración la variedad de dimensiones analizadas. Asimismo, en cuanto a la opinión de los estudiantes sobre las temáticas consultadas, se observa una tendencia a considerar que éstas no se incluyen, o se incluyen poco. Tanto el texto de la Ley, como el de los instrumentos internacionales en Salud Mental, toman la cuestión de la formación como nodal y se espera que el cambio sea paulatino y acompañado por la efectiva reforma de los servicios de salud mental. El resultado expuesto, servirá para comparar con futuras investigaciones, la implementación de los cambios.

Nos encontramos en un período de cambios en relación al paradigma de atención en Salud Mental, y la formación de profesionales es parte de dicho cambio. Por lo tanto, si bien se observan las mencionadas dificultades, debe considerarse el momento de transformación y transición por el cual se está atravesando. Tal como lo planteaba Kuhn (1971), hay circunstancias en las que pueden coexistir dos paradigmas en el último periodo.

En segundo lugar, se observa que en la bibliografía propuesta por las asignaturas, las temáticas Interdisciplina y Derechos $\mathrm{Hu}$ manos evidenciaron un alto peso en comparación con el resto, siendo las mismas de gran importancia en el marco de LSM. Pero, por otra parte se observa acuerdo en cuanto a la opinión de los alumnos, de que la inclusión de ambas temáticas, es escasa. En este sentido, se observa discrepancia entre lo que se incluiría formalmente en los pro- gramas y lo que los alumnos opinan respecto al proceso formativo que atravesaron o se encuentran atravesando.

En cuanto a la interdisciplina, es interesante destacar que observando la currícula de la carrera de Psicología en la UNMdP, se incluyen asignaturas referidas a diferentes disciplinas, y en este sentido se podría pensar en una formaión interdisciplinaria. Así, en el ciclo de formación básica encontramos Biología Humana; en el ciclo de formación general y complementaria, Sociología, Filosofía del Hombre, Antropología, Epistemología General.

Sin embargo, esto no garantiza una auténtica preparación de profesionales en las competencias necesarias para desempeñarse en equipos interdisciplinarios. La currícula presenta una pluralidad de asignaturas aisladas, pero esto no equivale a un diálogo y apertura de unas y otras. Por lo tanto, sería deseable que se creen espacios de discusión y debate sobre la forma en que esta temática debe ser transmitida al alumnado.

Respecto a la formación dentro del paradigma de Derechos Humanos, debe diferenciarse la transmisión del contenido de las declaraciones e instrumentos, y una formación que apunte al respeto de los derechos del usuario de los servicios de Salud Mental. De esta forma, el tipo de pensamiento que se busca fomentar en los futuros profesionales puede ser o bien de tipo "critico" o "explicador"; siendo lo que marca la diferencia entre uno y otro, lo mismo que diferencia una acción orientada a promover, garantizar y proteger los DDHH del normativismo abstracto de los DDHH (Perceval, 2011).

A partir de ambas fases del estudio, se observa coincidencia en lo que serían áreas poco desarrolladas en la formación del psicólogo en la UNMdP, en primer lugar la temática de la Psicofarmacología, la cual ha sido ampliamente discutida en el marco de la sanción de la LSM. Si bien la administración de Psicofármacos no es una incumbencia propia de los Psicólogos, para poder lograr que los tratamientos farmacológicos se den en el marco de abordajes interdisci- 
plinarios, es necesario contar con conocimientos mínimos en el área.

En cuanto a las temáticas de Consentimiento Informado e Historia Clínica, en el análisis de programas se observó escasez de ambas. Sin embargo, en opinión de los estudiantes el Consentimiento es incluido en la formación, mucho más que la cuestión de la confección y administración de Historias Clínicas. Estos aspectos son relativamente novedosos para los psicólogos, de forma que se espera que progresivamente, aumente su consideración en la formación.

La pregunta que surge es en qué medida la Universidad forma profesionales que respondan a las exigencias que marca la so- ciedad, la cual hoy en día en el ámbito de la Salud Mental, está atravesada por un proceso de transformación de sus servicios en base a un paradigma que promueve y protege los derechos de los usuarios.

Se espera que los aportes de la presente investigación sean significativos para poder analizar la situación de nuestra carrera en relación a la temática. La investigación busca representar un primer acercamiento diagnóstico a cuál es la situación en la carrera de Psicología en la UNMdP, para conocer la realidad desde la cual se parte y sobre la cual se construirán los nuevos aportes y desafíos.

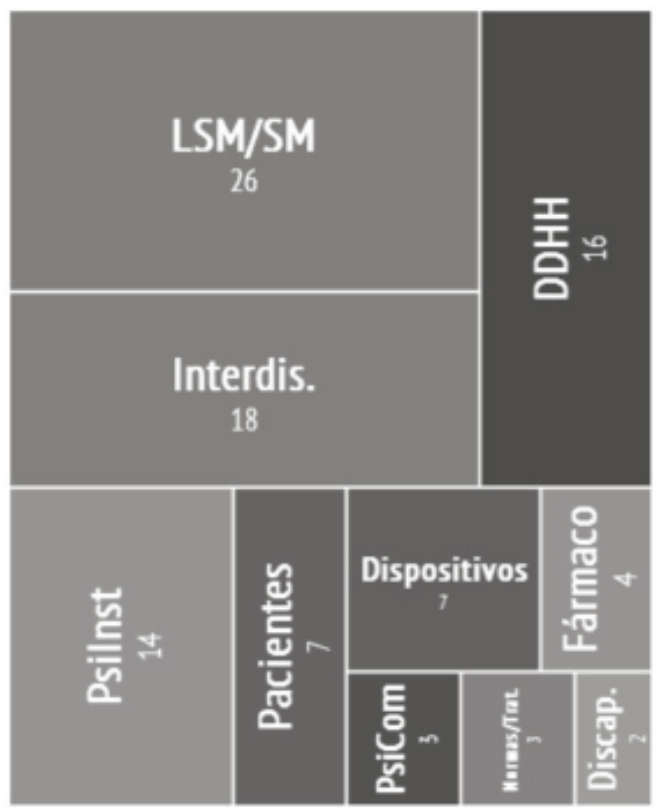

Nota:

LSM/SM: Ley de Salud Mental/Salud Mental

Interdis.: Interdisciplina

DDHH: Derechos Humanos

PsiInst.: Psicología Institucional

PsiCom.: Psicología Comunitaria

Discap.: Discapacidad

Normas/Trat.: Normas/Tratados

Figura 1. Peso expresado en porcentaje de las temáticas de acuerdo a la cantidad de referencias bibliográficas observadas 


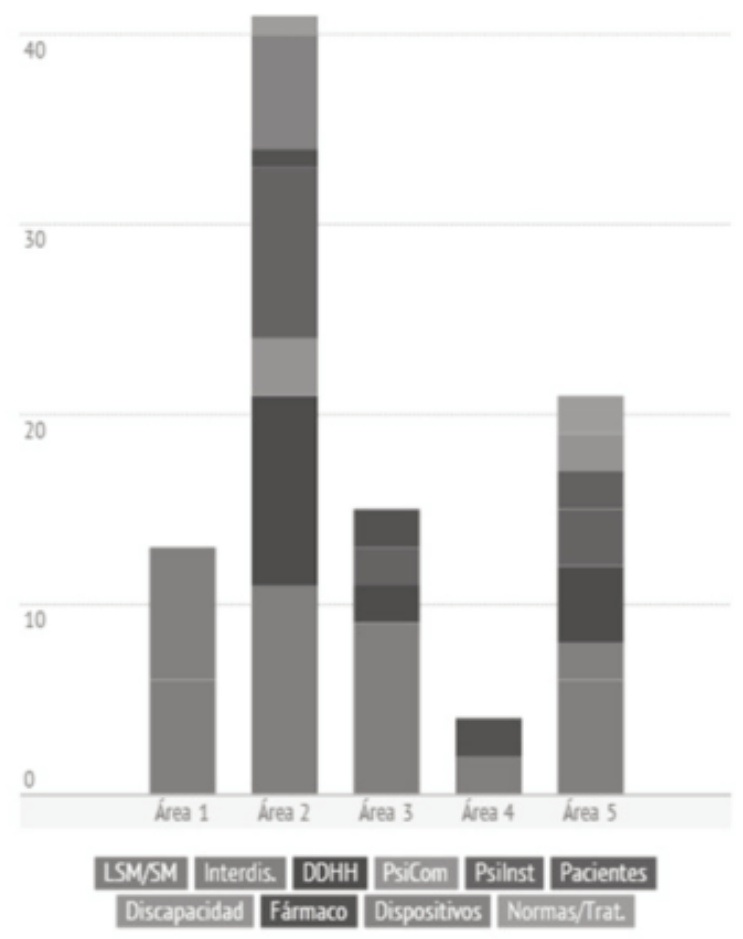

Figura 2. Distribución de las temáticas de acuerdo a la cantidad de referencias bibliográficas observadas por área de formación

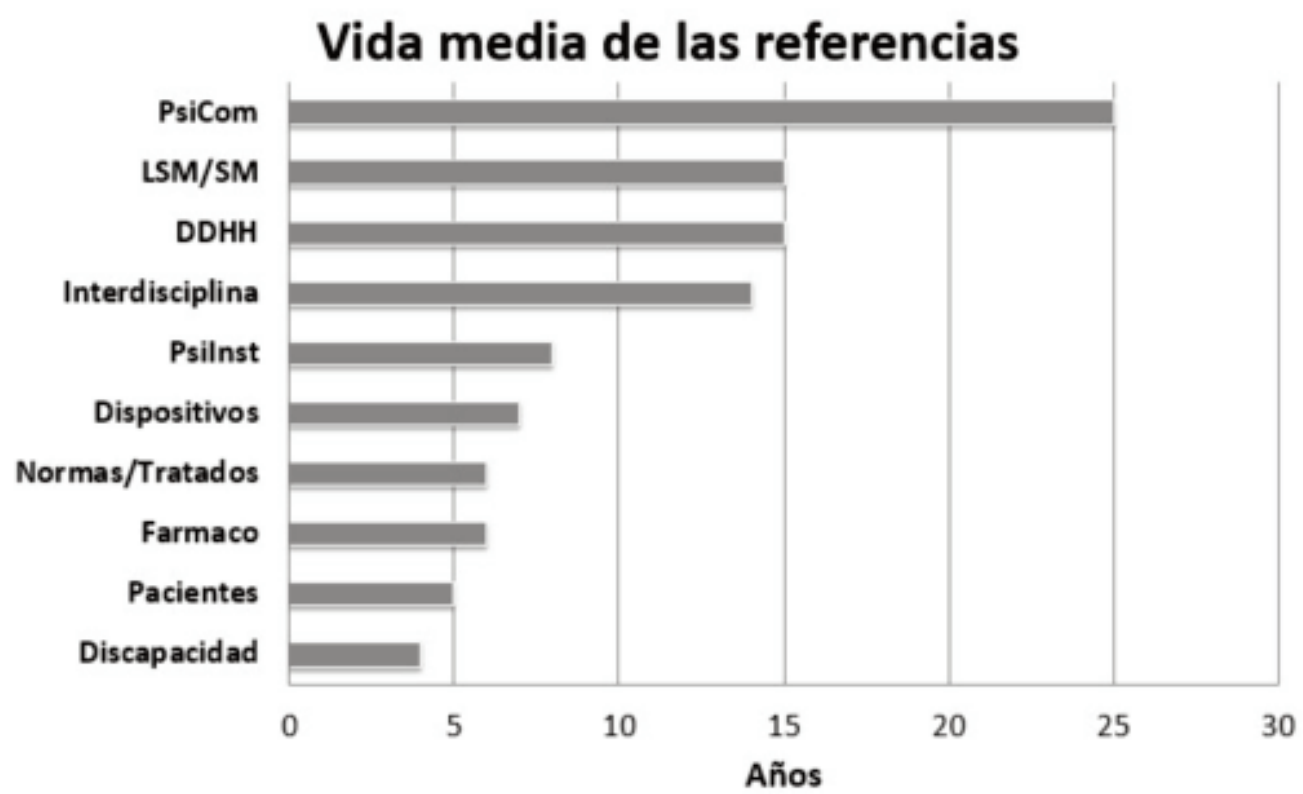

Figura 3. Vida media de las referencias 
Ley de Salud Mental 26.657: la formación de psicólogos

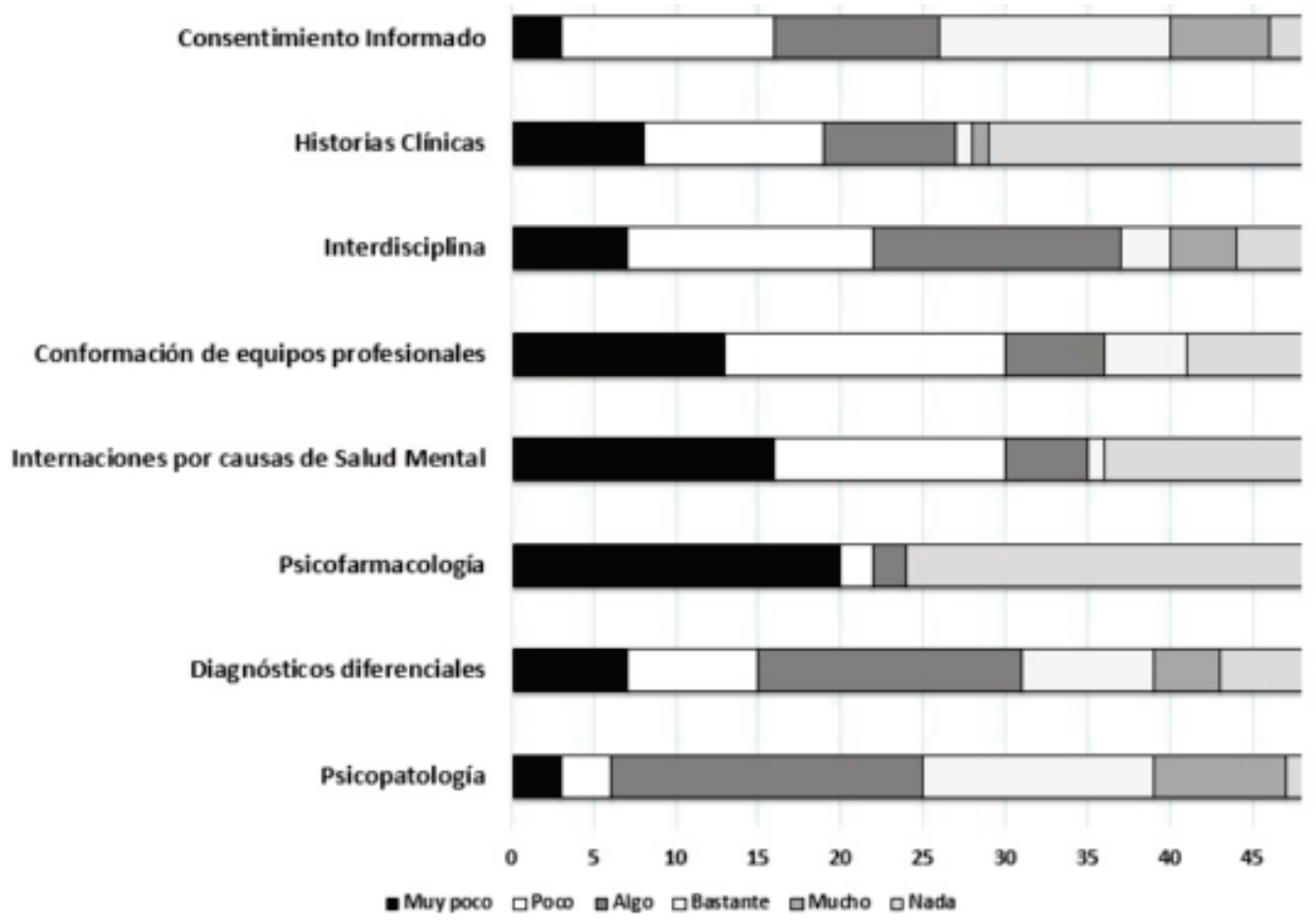

Figura 4. Opinión de estudiantes sobre la inclusión de temáticas en su formación

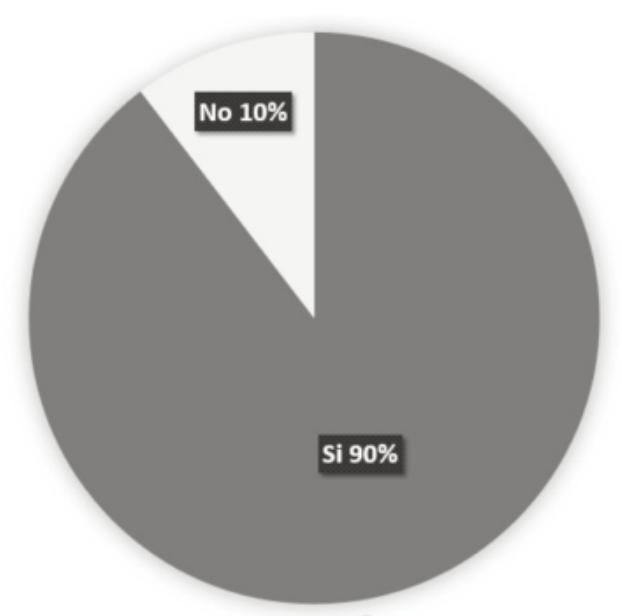

Figura 5. Opinión de los estudiantes sobre la inclusión en la formación de la cuestión de los Derechos Humanos 


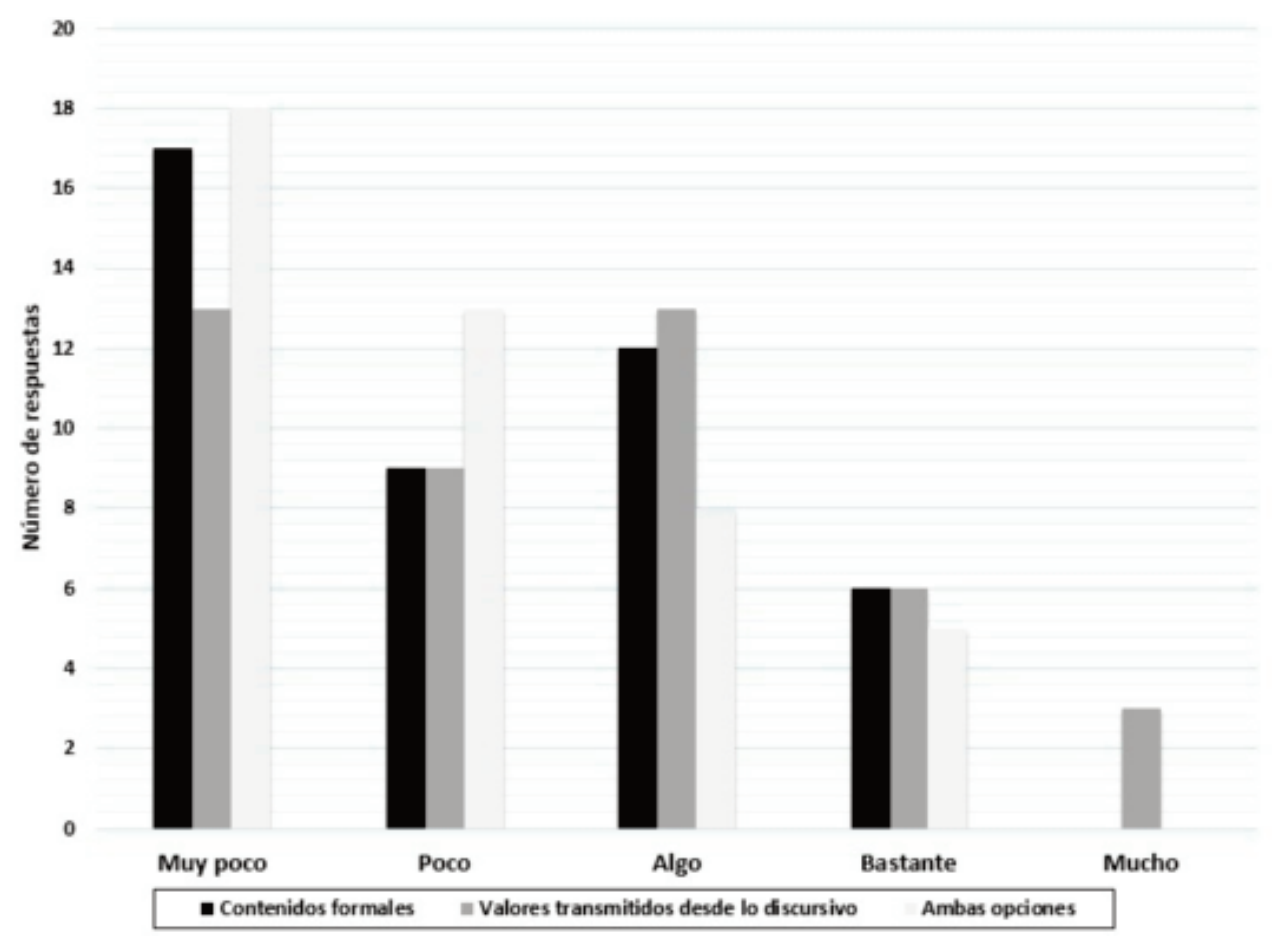

Figura 6. Opinión de los alumnos respecto a la forma de inclusión de la temática Derechos Humanos y a la medida en la cual se incluye

\section{Referencias bibliográficas}

Borgioli, M., Canchelara, L., De Uriarte, M.E., Guerra, M.M., Otegui Banno, M.M., Ponce, M., Rodríguez, C. \& Villa, P. (2011). El rol del residente psicólogo en el Hospital General a la luz de la Nueva Ley Nacional de Salud Mental. Ponencia presentada en el V Congreso Marplatense de Psicología. Consenso de Panamá, artículo 2, inciso F. Octubre 2, 2009.

D' Agostino, A.M.E. (2015). Salud mental ¿un contenido presente en la formación de grado? Un estudio en la facultad de Psicología, UNLP. Perspectivas en Psicología, 12(3), 15-25.

De Lellis, M. (2011). A propósito de la Ley Nacional de Salud Mental: nuevos escenarios y desafíos para la formación del psicólogo. Revista Diálogos. Universidad de San Luis, 2(2), 7-25.

Declaración de Caracas, artículo 5. Noviembre 11-14, 1990.
Hermosilla, A. \& Cataldo, R. (2014). Estudio exploratorio sobre la opinión de profesionales marplatenses sobre la Ley de Salud Mental. Revista Acta Psiquiátrica y Psicológica de América Latina, 60(2), 130-139.

Kuhn, T. (1971). La estructura de las revoluciones cientificas. México: FCE.

Ley Nacional de Salud Mental (26657/2010) Boletín Oficial N ${ }^{\circ} 32041$.

Más, F. (2010). Formación en psicología para el ejercicio profesional en salud pública. PSIENCIA: Revista Latinoamericana de Ciencia Psicológica, 2(2), 89-94.

Morán-Barrios, J. (2013). Un nuevo profesional para una nueva sociedad. Respuestas desde la educación médica: la formación basada en competencias. Revista de la Asociación Española de Neuropsiquiatría, 33(118), 385-405.

Perceval, M.C (Junio, 2011). Hacia una red interuniversitaria en Derechos Humanos. Trabajo presentado en la Jornada Nacional sobre Derechos Humanos y Universidad. 
Ciudad Autónoma de Buenos Aires. República Argentina. Ley Nacional No 26.657.

Restrepo-Espinosa, M.H. (2006). Promoción de la salud mental: un marco para la formación y para la acción. Revista ciencias de la salud, 4.

Rosendo, E. (Agosto, 2011). Desafios en la implementación de la Ley Derecho a la Protección de la salud Mental (Ley $N^{o}$ 26.657). Ponencia presentada en las IX Jornadas Nacionales de Debate Interdisciplinario en Salud y Población "Derecho a la salud y protección social". Facultad de Cien- cias Sociales, Universidad de Buenos Aires. Buenos Aires, Argentina.

Zaldúa, G., Bottinelli, M.M., Sopransi, M.B., Nabergoi, M., Lenta, M., Tisera, A., Freire, M. (Agosto, 2011). Cuestiones y desafios en la viabilidad de la Ley de Salud Mental. IX Jornadas Nacionales de Debate Interdisciplinario en Salud y Población, Área de Salud y Población. Instituto de Investigaciones Gino Germani. Buenos Aires, Argentina. Publicado en CD-ROM. ISBN: 9978-950-29-1322-3.

Recibido: 19 de noviembre de 2016 Aceptado: 14 de marzo de 2018 
